Revue d'histoire de l'Amérique française

REVUE D.HISTOIRE DE L'AMÉRIQUE FRANÇAISE

\title{
Le Canada aborigène dans le contexte historique
}

\section{Jacques Rousseau}

Volume 18, numéro 1, juin 1964

URI : https://id.erudit.org/iderudit/302341ar

DOI : https://doi.org/10.7202/302341ar

Aller au sommaire du numéro

Éditeur(s)

Institut d'histoire de l'Amérique française

ISSN

0035-2357 (imprimé)

1492-1383 (numérique)

Découvrir la revue

Citer cet article

Rousseau, J. (1964). Le Canada aborigène dans le contexte historique. Revue

d'histoire de l'Amérique française, 18(1), 39-63. https://doi.org/10.7202/302341ar d'utilisation que vous pouvez consulter en ligne.

https://apropos.erudit.org/fr/usagers/politique-dutilisation/ 


\section{LE CANADA ABORIGÈNE DANS LE CONTEXTE HISTORIQUE}

\section{QUI SONT LES AMÉRINDIENS ?}

Biologiquement, les Amérindiens ${ }^{1}$ sont de race mongolique, ce qui n'élimine pas la possibilité d'apports allogènes à l'époque préhistorique. Aux jours précolombiens, l'habitant de l'ouest de l'Amérique du Sud a pu absorber des éléments mélanésiens, et celui du nord-est, des éléments européens. La dominante reste asiatique, mais avec des traits européides nombreux.

Quand on découvrit que l'Amérique était un continent nouveau, séparé de l'Asie par l'océan Pacifique, on se mit à spéculer sur l'origine des peuplades autochtones du Nouveau-Monde. La plupart des auteurs modernes s'en tiennent aux migrations par le détroit de Behring. Le problème, cependant, reste à résoudre définitivement. Même si cette voie reste la principale, il n'est pas exclu que d'autres aient dû être empruntées à l'occasion. F. Ridley et Thomas E. Lee ont remis récemment en question la route transatlantique. Même d'origine mongolique des ancêtres des Amérindiens auraient pu gagner l'Amérique par l'Atlantique. Si cette hypothèse se justifiait, elle n'éliminerait pas pour autant les déplacements par le nord du Pacifique. Les migrations préhistoriques, sans plan défini, obéissaient seulement à la consigne de la faim; elles auraient pu se faire des deux côtés à la fois.

\section{DISTRIBUTION ET EVOLUTION DÊMOGRAPHIQUE DES AMÉRINDIENS DU CANADA}

Les recherches philologiques n'indiquent que des affinités tenues entre les langues asiatiques et amérindiennes. Des 160

${ }^{1} \mathrm{Le}$ terme amérindien en anglais amérindian ou amerind englobe tous les indigènes d'Amérique, Esquimaux compris, habitant l'Amérique avant la découverte, et leurs descendants. 
familles linguistiques, -1200 dialectes dans les trois Amériques, - deux ou trois seulement montrent une vague parenté avec des langues de l'Asie. L'athapascan renferme des traits sinotibétains et l'esquimau, des traits ouraliens. On groupe actuellement les langues amérindiennes, parlées au nord du Mexique, en 48 familles distinctes, groupées en six classes, sans aucune relation connue. Cette disparité linguistique suggère des migrations de hordes différentes, réparties sur plusieurs millénaires.

Onze familles linguistiques, réunies en quatre grandes classes, se partagent les Amérindo-canadiens. L'algique ou algonkienne ${ }^{2}$, représentée par la moitié des indigènes du pays $(115,900$ en 1961$)^{3}$, va de l'Atlantique au contrefort des Rocheuses. La famille iroquoise-huronne compte au Canada 20,000 sujets, pour la plupart en Ontario. L'athapascane dans le nord-ouest, et le salish, limitée aux Rocheuses, comprennent environ 18,000 personnes chacune. La famille esquimaude, qui a adopté le rivage arctique et une partie des territoires du nord-ouest, atteint 12,000 , sans compter les populations du Groenland $(28,000$ en 1962), de l'Alaska $(16,000$ en 1950) et du nord-est de l'Asie (1,100 en 1959). Dans la prairie canadienne, 4,400 habitants parlent des dialectes sioux. Les cinq autres familles, propres aux Rocheuses et à la côte du Pacifique, restent les moins importantes numériquement au Canada: le tsimshian et le wakashan, groupent les idiomes de 6,300 à 6,700 personnes chacun; les indigènes de langue Haida sont 1,150; enfin, les dialectes kootenay et tlinkit sont l'apanage de 440 personnes chacun.

On évalue la population indigène de l'Amérique, au nord du Mexique, à l'époque de la découverte, à 1,150,000 âmes, dont environ 220,000 pour le Canada. En 1910, elle se réduit à 403,000, pour le Canada et les États-Unis, mais depuis elle s'accroît. En 1961, base des statistiques présentées ici, le Canada en compte

2 La famille algique ou algonkienne (en anglais, algonquiam ou algonkian) comprend de nombreuses tribus, les Micmacs, Montagnais, Cris, Algonquins proprement dits (en anglais algonquin ou algonkin). Le mot algonkin, qui ne se distingue pas phonétiquement d'algonquin est à rejeter en français pour la famille.

${ }^{3}$ Les statistiques démographiques, sauf avis contraire, sont du recensement de 1961. 
environ 204,000 ce qui nous ramène à la population du XVIe siècle. Le recensement des Montagnais-Naskapi de la péninsule Québec-Labrador, qui illustre bien cette augmentation, les évalue à 3,800 en $1857,4,500$ en $1924,4,900$ en 1951 et près de 9,000 en 1961. Des tribus et des bandes, qui jouèrent un rôle important dans l'histoire du Canada, sont disparues ou réduites à la portion congrue: ainsi les Attikamek, Pétun, Neutres, Erié et Hurons. Les Beothuk de Terre-Neuve, éliminés par les pêcheurs anglosaxons de cette colonie, furent le seul peuple du Canada délibérément exterminé. ${ }^{4}$

Des jugements trop hâtifs attribuent à la guerre la décroissance numérique des Indiens. La plupart succombèrent d'abord aux maladies, nouvelles pour eux et apportées par les Blancs. Au début de son alliance avec les Français, la nation huronne comprenait, croit-on, 30,000 âmes contre 15,000 Iroquois. La rougeole sévit chez les premiers en 1634, suivie bientôt par la variole et une autre épidémie. En 1640, ils n'étaient plus que 12,000 quand le nombre des Iroquois restait sensiblement le même. La disparité épidémiologique s'explique. Les alliés hurons et algiques visitent régulièrement les établissements de la NouvelleFrance avec leurs familles et campent de longues semaines, en contact avec les colons, s'exposent ainsi à une contagion nouvelle. Les missionnaires et commerçants français, d'autre part, fréquentent librement leurs bourgades, tandis que les Hollandais et les Anglo-Saxons pénètrent plus rarement chez les Iroquois. Les Agniers vivent au cœur de la forêt; par de longs portages, leurs émissaires se rendent à Fort-Orange pour de brèves transactions. Des marcheurs isolés, pressés de retourner, évitent mieux la contamination que des maisonnées entières venues en canots et qui s'attardent. La famille iroquoise n'est pas la bienvenue à Fort-Orange et cela l'a sauvée. Après 1643, les Hollandais fournissent des armes aux Iroquois, mais les Français, craignant la révolte, refusent d'en munir leurs alliés non chrétiens. En 1649, quand les Cinq-Nations donnent le coup de grâce aux Hurons, la maladie les avait déjà vaincus avant. L'histoire accarouges.

4 James P. Howley (1915). - Jacques Rousseau, Le Dernier des Peaux 
ble souvent les victimes. En vérité, les Hurons égalaient les Iroquois; les uns et les autres sont de même race, de même langue, de même caractère et de même structure sociale. N'oublions pas que les Iroquois avaient l'avantage de l'agression. Les Hurons, placés sur le chemin de la fourrure au nord, pouvaient servir d'intermédiaires entre les chasseurs algiques et les Blancs. Ils n'avaient aucun intérêt à attaquer l'Iroquois, mais celui-ci, moins bien pourvu pour le commerce nouveau se devait de harceler le compétiteur ou adversaire plus heureux.

A côté des pertes, le Canada fait des acquisitions. Alliés des Français, fuyant l'ennemi anglophone, des Abénaquis du Maine vinrent s'établir en 1700 sur le Saint-Laurent. Des loyalistes iroquois, fidèles à la couronne anglaise, émigrent en Ontario lors de l'indépendance américaine. Derniers venus en territoire canadien, des Sioux rebelles quittent les États-Unis, en 1876, pour chercher asile dans la Prairie canadienne, avec leur chef Sitting Bull. Attiré dans un guet-apens au-delà de la frontière on l'abattra en 1890: l'histoire du duc d'Enghien se répétait.

Rien n'illustre mieux le dynamisme de la population amérindienne que l'histoire de l'occupation de la vallée du Saint-Laurent. Sur les habitants du territoire, un demi-millénaire avant l'arrivée des Blancs, nous sommes réduits à des conjectures basées sur des pointes de flèches et de rares ossements. Nous savons peu de choses du chasseur pré-algique, l'Homme de l'ocre rouge. Peut-être s'identifie-t-il aux Béothuk, rencontrés à Terre-Neuve par les premiers navigateurs et que l'on a improprement appelés Peaux-rouges, parce qu'ils s'enduisaient la figure d'ocre. Premier occupant connu de la vallée du Saint-Laurent, reliant les lacs avec le canot, l'Algique avait maîtrisé la forêt boréale, où se dressait au gré des chasses sa cabane légère. Pendant ce temps, l'agriculture naissait de la maîtrise de l'eau sur les plateaux arides du sud. Quelque part sur l'Ohio, une bande reçut un jour des dieux le maïs, le haricot, la courge et le tabac. Liés par des modes de vie qui subordonnent l'individu à la collectivité, les Iroquois perfectionnent leur organisation sociale et fondent la Ligue des Cinq-Nations, qui jouera plus tard un rôle déterminant dans les destinées du continent. A l'affût de terres à cultiver, de 
conquêtes en conquêtes, ils atteignent le Saint-Laurent qu'ils colonisent au moins jusqu'à Québec et en délogent les bandes algiques. L'appauvrissement du sol oblige le village à déménager tous les trente ans. Entre les voyages de Cartier et de Champlain, l'Iroquois se replie à l'ouest des rapides de Lachine et s'y installe définitivement, pendant que l'Algonkien reprend une partie de son ancien territoire. Le Blanc entre alors en scène et tente de reconstituer en terre étrangère ses paysages familiers de l'ouest de la France.

L'Amérindien a une répartition géographique fluctuante. Essayons de nous le représenter dans l'est du pays, au milieu du XVIIe siècle quand naquit la colonie française. ${ }^{5}$

Esquimau. (Jusqu'aux environs de 1900, les auteurs anglais écrivaient de préférence esquimau, le mot français, tiré lui-même d'un vocable montagnais-naskapi; mais depuis, la forme germanique et scandinave eskimo a prévalu.) Ces indigènes, qui se nomment eux-mêmes Inuit, - c'est-à-dire "les hommes, les vrais hommes", - appartiennent à une famille linguistique distincte, l'esquimaude. Dans la péninsule Québec-Labrador, ils sont partagés en nombreux groupements, indistincts culturellement et linguistiquement, sauf parfois par des nuances idiomatiques et des traits écologiques. Ils occupent aujourd'hui le littoral arctique de la péninsule, depuis les environs de Hamilton Inlet, sur la côte du Labrador, jusqu'au nord de la rivière Fort-George, sur la baie James. Au début de l'époque historique, ils ont atteint Havre-Saint-Pierre (autrefois Pointe-aux-Esquimaux), sur la côte nord du Saint-Laurent. Les relations des anciens voyageurs mentionnent des contacts fugaces entre Blancs et Esquimaux. Dans la recherche du passage du Nord-Ouest, ils ont joué un rôle d'obstruction par leur hostilité aux Européens. Les relations cordiales, sur la côte du Labrador, débutent avec leur évangélisation par les Frères Moraves. Sur le reste du littoral, leurs échanges commerciaux avec les Blancs, épisodiques depuis environ un siècle, ne s'établissent vraiment qu'à la veille du

5 Pour l'énumération des tribus et bandes, on procédera du nord au sud et de l'est à l'ouest. - Voir notamment Jenness, op. cit. et Rousseau $(1958,1959,1960)$. 
XXe siècle. Comme l'ont révélé des voyages d'explorations de l'auteur, accompagné par Jean Michéa, des Dorset (rattachés habituellement aux Esquimaux) occupèrent la région du lac Payne, à l'intérieur de l'Ungava, et y édifièrent un établissement permanent fait de nombreuses maisons et pierres carrées. Leur disparition remonte peut-être à plusieurs siècles. Que les indigènes de la culture Dorset soient de la civilisation esquimaude reste à démontrer. Il est même possible qu'ils soient apparentés aux Béothuk.

Béothuk. Appartenant probablement à une famille linguistique distincte, les Béothuk comptaient une population d'environ 500 âmes, établie entièrement à Terre-Neuve, à l'époque de la Découverte. Pourchassés par les colons anglais, les rares survivants disparurent au milieu du siècle dernier, alors que des gouvernants dans un tardif sursaut de conscience tentèrent de les sauver. Jamais aucun Blanc n'avait tenté d'apprendre leur langue et aucun missionnaire anglo-saxon ne les visita jamais. ${ }^{6}$

Montagnais-Naskapi. ${ }^{7}$ De la famille linguistique algique ou algonkienne et comprenant plusieurs dialectes rapprochés. Il n'est pas facile de distinguer nettement Naskapi et Montagnais, tant il y a d'éléments de transition. D'ailleurs, les mariages et les adoptions n'aident guère à clarifier le problème. Dans le présent cas, la meilleure ligne de démarcation est un trait culturel: chasseurs migrateurs de la forêt tempérée les Montagnais poursuivent l'orignal et le castor au nord de la vallée du Saint-Laurent et à l'est de la rivière Saint-Maurice; tandis que les Naskapi sont des nomades attachés aux hordes de caribous des zones subarctique et hémiarctique. La répartition des bandes peu avant 1900 nous donne idée de leur distribution deux siècles plus tôt; mais il y eut parfois regroupement. Vers la fin du siècle dernier les Montagnais-Naskapi comptaient les bandes suivantes: 1. Naskapi : a) Barren Ground (rivière George), disparus vers 1940-1945, comme l'ont révélé les voyages d'exploration de l'au-

${ }^{6}$ Sur les Béothuk, voir notamment Howley, op. cit., et Rousseau, Le dernier des Peaux-rouges.

7 Sur les Montagnais-Naskapi du Québec, voir Speck, op. cit. et les diverses publications de Jacques Rousseau, citées à la bibliographie et qui donnent les sources principales. 
teur; Davis Inlet, Northwest River (celle-ci comprenait des Naskapi et Montagnais) à l'est de la péninsule; b) Ungava, Petitsikapau, Michikamau et Kaniapiskau, au centre de la péninsule; c) Rivière de la Baleine-blanche (White Whale River band), Grande Rivière à la Baleine (Big River band), sur le versant occidental de la péninsule. - 2. Montagnais-Naskapi intermédiaires: Nichikoun, Eastmain, Mistassini et Waswanipi, au centre et à l'ouest de la péninsule. - 3. Montagnais proprement dits (toutes les familles occupant le nord du territoire, entre Saint-Augustin et Sainte-Marguerite, sont souvent d'affinité naskapi): Saint-Augustin, Musquaro, Natashquan, Mingan, Moisie, Ste-Marguerite, Sept-îles, Godbout, Bersimis, Escoumains, Tadoussac, Chicoutimi, Lac Saint-Jean, - 4. Montagnais-Moskégon de l'ouest de la province (Rupert House, Moosonee), intermédiaires entre les Montagnais et les Moskégon dont il sera question plus loin. - Dans la terminologie anglaise, Naskapi et Montagnais-Naskapi de l'ouest du Québec reçoivent souvent l'appellation de Cree, qui devient alors une source de confusion. Ceci s'explique par le fait que les Cris de l'Ouest et Montagnais-Naskapi sont étroitement apparentés au point de vue linguistique. Les historiens pourront s'étonner de ne pas voir plus haut la mention du "peuple" des Papinachois, malgré son importance dans les récits des anciens missionnaires, qui les situaient à la hauteur des terres entre le territoire de Mistassini et le Labrador. Leurs descendants, probablement, font partie des bandes de la Côte-Nord du St-Laurent. Après l'établissement de la traite des fourrures les indigènes de l'intérieur durent trouver un débouché vers la mer. Les Mistassins, pour la même raison, évoluèrent longtemps entre leur pays et Rupert House. On comprend que le peuple papinachois ait pu se fragmenter, suivant les bassins hydrographiques, et s'allier aux Montagnais du cours inférieur des rivières. Les Papinachois, en quelque sorte, seraient les ancêtres des Montagnais-Naskapi chassant aujourd'hui à la hauteur des terres et ayant leurs attaches commerciales et sociales au bord du Saint-Laurent.

Algonquin. (En anglais, Algonkin ou Algonquin). De la famille linguistique algique ou algonkienne (en anglais, algon- 
quian ou algonkian). Le territoire des Algonquins, dans le Québec, - à l'ouest du St-Maurice et au sud de la ligne de partage des eaux, - englobait tout le bassin hydrographique de l'Outaouais. Les statistiques d'avant 1894 en plaçaient des groupes résiduels au Cap-de-la-Madeleine, où ils avaient un centre important sous le régime français. Aujourd'hui, dans le Québec, on en rencontre à Oka (avec des Iroquois), Maniwaki et rivière Désert sur la Gatineau, île aux Allumettes (rivière Outaouais), rivière et lac Dumoine, Pontiac et Témiscamingue, région de Mattawa (Témiscamingue) lac des Quinze (Témiscamingue), lac Barrière (Nord du lac de Pontiac), Grand Lac Victoria (Témiscamingue). Au sud de l'Outaouais, en Ontario, Golden Lake, North Renfrew et Gibson (en partie iroquois). Dans l'ensemble, ils occupent donc le bassin de l'Outaouais et le sud de l'Abitibi.

Attikamek (ou Attikamègues ou Poissons-blancs, équivalent français du nom indigène), de la famille algique. Vivant dans la partie supérieure du St-Maurice, au début du régime français, décimés d'abord par la variole, ils furent tellement harassés par les incursions iroquoises au milieu du XVIIe siècle qu'ils disparurent comme groupement distinct vers 1670, les résidus se réfugiant chez les Montagnais de Tadoussac. Nous ne savons pas s'il faut les rattacher aux Montagnais ou aux Algonquins, ou même s'ils constituent un groupement distinct, peut-être apparenté aux Tête-de-boule.

Tête-de-boule. De la famille linguistique algique, habitant la hauteur des terres entre le bassin du St-Maurice et celui de la baie James, et comprenant les bandes de Manouan (lac Kempt), Weymontachingue et Obidjuan (près de Oskalaneo). Les Têtesde-boule, plus apparentés aux Ojibway qu'aux Montagnais, sont peut-être de nouveaux venus dans le nord du Québec. Leurs récits traditionnels, qui font état de la "peur séculaire de l'Iroquois", 8 laissent croire cependant qu'ils habitaient déjà un territoire entre l'Outaouais supérieur, le sud de l'Abitibi et le haut SaintPierre, quand les Iroquois ravagèrent ces régions au milieu du XVIIe siècle.

8 Rousseau et Rousseau, op. cit. 
Micmac. (Étymologiquement, "les alliés"). Nommés fréquemment Souriquois dans les vieilles relations, de la famille linguistique algique, les Micmacs occupèrent la Gaspésie, les deux tiers du Nouveau-Brunswick (côté oriental), la nouvelle-Écosse et l'Île-du-Prince-Édouard au complet. Après l'époque des découvertes, ils envahissent des secteurs de Terre-Neuve, où ils pourchassent les Béothuk avec la complicité des Blancs. Dans le Québec, iis habitent aujourd'hui Ste-Anne-de-Ristigouche et Maria.

Malécite. Nommés souvent Etchemins dans les vieilles relations et faisant partie de la famille linguistique algique, les Malécites habitent un territoire allant du Saint-Laurent, dans le comté de Témiscouata, jusqu'à la partie inférieure de la rivière StJean (Maine et Nouveau-Brunswick). Le Québec n'en compte plus qu'une petite réserve dans le comté de Témiscouata et quelques familles dispersées, entremêlées d'Abénaquis et complètement assimilées, dans la région de Bécancour.

Abénaquis. De la famille algique et autrefois d'une confédération du Maine, les Abénaquis du Québec descendent de réfugiés fidèles aux Français et pourchassés du nord-est des ÉtatsUnis en 1700. Établis à St-François-du-lac, sur la rive sud du St-Laurent, ils se livrèrent à une agriculture marginale sur les terres mises à leur disposition et reçurent pour la chasse la région occupée autrefois par les Attikamek ou les Algonquins, au voisinage de St-Michel-des-Saints, au nord du fleuve, et devenue vacante.

Moskégon. (En anglais, Swampy Cree ou Muskegon, dérivant de Muskeg, marécage dans plusieurs dialectes algiques). De la famille linguistique algonkienne, occupant les terrains marécageux à l'ouest des baies James et Hudson, au sud de Churchill, et en général tout le bassin de ces nappes d'eau en Ontario. On a confondu sous le nom anglais de Cree (dérivé du français Cri ou Cris, féminin Crise, lui-même une abréviation de vieux français Kristineau), de nombreuses bandes, depuis le lac Mistassini jusqu'à la prairie de l'ouest et aux territoires du NordOuest. Le fait que les commerçants de fourrures pouvaient uti- 
liser la même langue (ou presque) dans cet immense territoire engendra cette confusion onomastique. Les prétendus Cris du nord du Québec, versant oriental de la baie d'Hudson, sont des Montagnais-Naskapi; ceux qui vivent sur les terrains bas et marécageux, à l'ouest des baies James et Hudson, sont les Moskégons. Quant aux bandes des territoires du Nord-Ouest et des forêts du nord des provinces centrales (rivières de la Paix et Athabaska, lac Athabaska, lac des Esclaves et Grand lac de l'Ours), elles se distinguent assez des Moskégons sur le plan culturel pour mériter un nom particulier. A défaut de mieux, nous pouvons les nommer Cris forestiers du Nord-Ouest. Enfin, les Cris de la plaine diffèrent de tous les précédents par leur régime de vie.

Outaouais (en anglais, Ottawa Indians). De la famille algique, assez rapprochés des Ojibway et des Potawatomi, ces trois tribus, suivant leurs traditions auraient une origine commune. Le nom Outaouais, dérivé d'un terme algique signifiant "trafiquer", s'explique bien: ces indigènes habitant primitivement la baie Georgienne, l'île Manitoulin et le voisinage de Michillimakinac, servaient d'intermédiaires commerciaux entre les diverses peuplades des Grands Lacs et, par la rivière Outaouais qu'ils considéraient leur artère, entre ces dernières et celles du Québec. Les Cheveux-relevés, rencontrés par Champlain en 1615 près de l'embouchure de la rivière des Français, dans la baie Georgienne, étaient des Outaouais. Marchands plutôt que guerriers, malheureux dans leurs combats tribaux, ils abandonnèrent l'île Manitoulin pour se disperser autour des Grands Lacs, mais une partie revint plus tard.

Huron. (Nom français dérivé de Hure, allusion à la chevelure réduite à une touffe. De la famille linguistique huronneiroquoise (en anglais, iroquoian family) et occupant le nord de la péninsule ontarienne, au sud de la baie Georgienne, la ligue huronne se nommait elle-même la Confédération des Wendat (étymologiquement "habitants de l'île ou de la presqu'île"), d'où le nom de Wyandote adopté par leurs descendants aux ÉtatsUnis (en Oklahoma, Michigan et Kansas). Elle comprenait quatre tribus différentes, celles de Attignaouantan (nation de 
l'Ours), des Attigneenongnahac (nation de la Corde), des Arendahronon (nation du Rocher) et des Tahontaenrat (nation du Cerf), chacune occupant plusieurs villages. La Confédération réunissait aussi de petites peuplades de même affinité linguistique et également une bande algique cherchant protection contre l'Iroquois. Décimés d'abord par des épidémies successives, quand la population huronne devint inférieure à celle des Iroquois, ces derniers, avec des armes hollandaises, les réduisirent à des lambeaux qui vinrent échouer aux États-Unis et au voisinage de Québec. Les Iroquois en adoptèrent aussi un grand nombre qui devinrent, conformément à la pratique courante, des sujets à part entière. Chez les Onondaga, en 1653 , le père Lemoine en rencontra 1000, entièrement intégrés à la tribu. Aux 500 ou 600 , établis sur l'île d'Orléans depuis 1649, les Iroquois présentèrent un ultimatum vers 1654: "Ou la fusion ou la mort ?" Le plus grand nombre alla remplacer dans des tribus iroquoises les guerriers tués à la guerre. Si bien que les Iroquois des Cinq-Nations, aujourd'hui, sont plus justement des Hurons-Iroquois. Il est à présumer que les Iroquois attaquant Dollard au Long-Sault en 1660 comptaient un certain nombre de Hurons adoptés, et cette perspective permet de mieux comprendre des phases du combat.

Iroquois. (Nom dérivant d'un mot algique signifiant "serpent"). De la famille huronne-iroquoise. La confédération iroquoise des Cinq-Nations comprenait cinq tribus, portant aujourd'hui les noms de Mohawk (anciennement Agnier, chez les Français), Oneida (anc. Onéïout), Onondaga (anc. Onondagué), Cayuga (anc. Goyogouin), Seneca (anc. Tsonnontouan). Vers 1715 , une autre tribu iroquoise, celle des Tuscarora, entra à son tour dans la ligue, devenue celle des Six-Nations.

Pétun. (En anglais, Tobacco Indian). En 1640, groupe de neuf villages indigènes étroitement apparentés aux Hurons (d'où le nom de Huron du Pétun dans les vieilles relations). Habitant la péninsule ontarienne, au sud-ouest des Hurons, ils n'entrèrent pas dans leur ligue pour rester indépendants dans les conflits hurons-iroquois, mais ils subirent néanmoins le même sort en 1649 . 
Neutres. (En anglais, Neutral). Confédérations de villages, au nombre de 40 en 1640 avec une population de 12,000 d'après Brébeuf. Peuple identique à celui des Hurons et des Pétuns, les Neutres vivaient entre ces derniers et les Iroquois, constituant ainsi un état-tampon. Leur neutralité fut respectée pendant les guerres huronnes-iroquoises, mais à la fin de celles-ci, en 1649, les Iroquois les harcelèrent au point qu'il n'en restait plus que 800 personnes en 1653. Les survivants se sont fusionnés avec d'autres tribus.

La plupart des peuplades amérindiennes de l'ouest du Canada n'ont rencontré les Européens qu'après les voyages de La Vérendrye. Il n'y a donc pas lieu de préciser ici leur distribution. On trouvera toutefois un aperçu plus loin.

Pour situer les problèmes historiques des XVIIe et XVIIIe siècles, il importe de mentionner rapidement les peuplades indigènes du nord-est des États-Unis, en bordure de la frontière canadienne, jusqu'au lac Supérieur et le long du Mississipi.

Maine (tel que délimité depuis le traité d'Ashburton). Renfermait des bandes algiques: Passamaquoddy près de l'embouchure de la rivière Saint-Jean (Maine et Nouveau-Brunswick), Malécites, puis vers l'ouest, Pénobscot et Abénaquis. Le territoire des Malécites, au nord de celui des Passamaquoddy, se rendait jusqu'au Saint-Laurent; ceux des Pénobscot et des Abénaquis dépassaient au nord la frontière actuelle du Canada, couvrant probablement une partie du massif alléghanien, dans les cantons de l'Est. Le pays des Pennacook, au sud-ouest du Maine, n'atteignait pas le territoire canadien.

New-Hampshire: Abénaquis et Pennacook. - Vermont: Abénaquis, Pennacook et Mahican, également algiques. Les Mahicans ou Mohicans (dont le nom signifie Loup dans les dialectes algiques) occupaient la partie supérieure, de la rivière Hudson et le lac Champlain, débordant probablement les limites actuelles du Québec.

New-York et New-Jersey. 1) Des Algiques: Delaware (île Manhattan et New-Jersey), Mahican et Montauk; 2) des tribus huronnes-iroquoises: Iroquois, Érié, Neutres, Tuscarora et au- 
tres. - Pennsylvanie: des Algiques (de la tribu des Delaware) et des Hurons-Iroquois (Érié, Iroquois, etc.).

Michigan et Wisconsin: Des Hurons-Iroquois (Hurons et Neutres) ; des Algiques (Ojibway, Renards, - anglais Fox, Menominee ou Folle-Avoine, Miami, Outaouais, Potawatomi, Illinois, Kickapoo, Sauk); et des Indiens de la famille sioux (Winnabago). - Minnesota: des Algiques (Ojibway et Cheyenne). - Ohio, Indiana et Illinois: des Hurons-Iroquois (Érié) et des Algiques (Delaware, Ojibway, Illinois, Miami, Shawnee, Outaouais et Potawatomi). - Iowa et Missouri: familles algique (Ojibway, Illinois) et sioux (Dakota, Iowa et Missouri). Kentucky et Tennessee: familles iroquoise (Cherokee), algique (Shawnee) et muskogéenne (anglais, muskogean) (Chickasaw, Kaskinampo, Chiaha, Tali). - Arkansas: familles algique (Illinois), iroquoise (Cherokee), caddo (Caddo), muskogéenne (Choctaw, Chickasaw, Kaskinampo), sioux (Quapaw), tunica (Tunica et Yazoo). - Mississipi: familles sioux (Biloxi), tunica (Koroa), muskogéenne (Chackchiuma, Choctaw, Houma, Pascagoula) et natchez (Natchez). - Louisiane: familles caddo (Adai, Natchitoches), natchez (Natchez), tunica (Atakapa, Chawasha, Chitimacha, Washa, Tiou, Tunica, Yazco) et muskogéenne (Acolapissa, Bayogoula, Choctaw, Okelousa, Quimpissa, Taenso, Tangipahoa).

Les indigènes du Maine, du New-Hampshire et du Vermont et des régions limitrophes du Canada sont à l'origine des “captivités" qui affectent la généalogie des citoyens de la NouvelleAngleterre et de la Nouvelle-France. Les Amérindiens de l'état de New-York furent surtout les artisans des guerres iroquoises. Ceux du voisinage des Grands Lacs, à l'ouest du lac Érié, et ceux du bassin du Mississipi prennent place dans l'histoire des découvertes et du commerce de la fourrure; ce pays, d'ailleurs, faisait alors partie de la Nouvelle-France.

\section{3. ÉVOLUTION DE LA CULTURE ET DE LA VIE ÉCONOMIQUE INDIGENES}

Le commerce des fourrures, - pendant longtemps l'axe de l'histoire du Canada, - s'effectuait entièrement en pays algique. 
A cause de cela, il s'imposait que Champlain fasse alliance avec les Hurons, - sur le chemin de la traite, - plutôt qu'avec les Iroquois, vivant au sud, et d'ailleurs beaucoup moins puissants ${ }^{9}$. Le mode de vie n'est pas lié cependant à la famille linguistique. Les Esquimaux sont typiquement des chasseurs maritimes, mais ceux du Caribou à l'ouest de la baie d'Hudson, vivent comme les Montagnais-Naskapi de l'hémiarctique québecois. La forêt retient la plupart des bandes algiques, sauf les Pieds-Noirs et les Cris de la Plaine, des chasseurs de bison. Les Athapascans chassent dans la forêt boréale de l'ouest (surtout subarctique et hémiarctique), sauf les Sarcis de la Prairie, liés au bison, et les Navajos du sud-ouest des États-Unis, vivant de rapine avant de devenir de paisibles pasteurs de moutons. Les Haïda et leurs voisins, les Tsimshian, de la côte du Pacifique, ont la même vie culturelle, mais appartiennent à des familles linguistiques sans parenté connue.

Chez les peuples amérindiens, pas plus que chez les peuples aryens d'ailleurs, l'affinité linguistique n'implique nécessairement un commerce amical. Irréductibles ennemis des Iroquois, leurs frères par la langue, les Hurons s'entendaient mieux avec les Algonquins, parlant un dialecte sans parenté évidente.

L'unité sociale et politique des primitifs, - habituellement la tribu, - consiste en un groupement de personnes possédant à la fois une communauté géographique, idiomatique et culturelle. Les tribus les mieux organisées, - celles des Iroquois et des peuplades de la côte du Pacifique, - se divisent à leur tour en phratries et en clans. Par contre, celles qui sont dispersées sur de vastes territoires se réduisent en bandes, habitant chacune un bassin hydrographique particulier, et auxquelles se ramène l'unité sociale et politique. Le peuple des Montagnais constituait une tribu au point de vue culturel et linguistique, mas purement théorique et sans gouvernement central, l'autorité se partageant entre les bandes des Escoumains, de Bersimis, des Sept-Îles, du lac Saint-Jean, etc. Unités assez ouvertes, toutefois, on pouvait passer facilement de l'une à l'autre, par le mariage surtout.

9 Jacques Rousseau, "Les premiers Canadiens", 50-51. 
Les manuels présentent souvent les indigènes comme des nomades. Beaucoup l'étaient à la façon des ouvriers et des fonctionnaires qui se déplacent suivant les exigences du métier ou de la vie économique. Même les vrais nomades, - comme les chasseurs de bisons ou de caribous, - évoluaient entre des bornes qu'ils ne pouvaient dépasser sans encourir le déplaisir des voisins. D'autres indigènes, - et beaucoup plus nombreux - avaient un port d'attache où ils revenaient périodiquement. Il y avait enfin de véritables villageois. Tenant compte de ces facteurs, nous pourrions ramener les Amérindiens du Canada, au début de la période historique, à six stades culturels différents.

1. Les chasseurs nomades du littoral arctique, les Esquimaux, vont de leurs camps d'été aux camps d'hiver, qui se déplacent suivant les caprices du gibier marin.

2. Les nomades de la forêt subarctique et hémiarctique, le Naskapi dans le Nord du Québec, l'Athapascan dans le Nordouest, - se plient aux migrations des hordes de caribous auxquelles ils s'attachent comme des parasites.

3. Les nomades de la plaine, - Sarcis (des Athapascans), Pieds-noirs, Gros-ventre et Cris (des Algiques), Assiniboine (de la famille sioux), - suivent servilement les troupeaux de bisons. Des Sioux proprement dits, venus d'outre-frontière, ont émigré au Canada au cours du siècle dernier.

4-a. Les tribus migratrices de l'est du Canada, - toutes de la famille algique: Micmacs, Malécites, Montagnais, Algonquins proprement dits, Ojibway, Mistassins, Cris, - et les autres bandes, également algiques, bordant la frontière du nord-est des États-Unis, vivaient exclusivement de la forêt. L'automne, les familles se dispersaient dans les territoires de chasse, chacune à vingt-cinq ou cinquante milles de la voisine, puis regagnaient au printemps le centre social. Les Béothuk, les vrais Peaux-rouges, habitant Terre-Neuve à l'époque de la Découverte, — et sur lesquels on sait peu de chose, - semblent des intermédiaires entre le migrateur de l'est et le nomade du littoral arctique. On peut même se demander si les Dorset, - connus uniquement par leurs 
restes préhistoriques et rattachés souvent aux Esquimaux, ne seraient pas apparentés aux Béothuk.

4-b. Les chasseurs migrateurs de la Cordillère, - comprennent des peuplades de langues tlinkit (les Tagish), athapascane (les Chilcotin, Porteurs, - en anglais Carriers, - Tsetsaut, Tahltan), salish et kootenay, vivent un peu comme les Montagnais.

5. Les agriculteurs semi-sédentaires hurons-iroquois habitaient des villages au voisinage des Grands Lacs et du St-Laurent, en amont de Montréal, à l'époque de Champlain: mais, lors des voyages de Cartier, ils se rendaient jusqu'à Gaspé. Se basant uniquement sur le vocabulaire accompagnant les relations de Cartier, on ne peut attribuer à des bandes iroquoises ou huronnes particulières l'occupation du St-Laurent. L'adoption était si fréquente alors qu'il est impossible de tirer des conclusions d'un vocabulaire recueilli peut-être chez deux individus seulement. De tous les Amérindiens de l'est, les Hurons-Iroquois ont atteint le plus haut degré de civilisation. Les Algonquins voisins des $\mathrm{Hu}-$ rons se sont parfois livrés à une culture rudimentaire du maïs, toujours marginale, cependant. La terre brûlée devenant inculte après 15 ou 20 ans de culture, on déménageait le village.

6. Enfin, les Amérindiens semi-sédentaires de la côte du Pacifique, - des familles linguistiques tlinkit, haïda, tsimshian, salish (les Bella-Coola et les Salish côtiers, séparés l'un de l'autre par les Kwakiutl), wakashan (comprenant les Kwakiutl et Nootka), - habitaient des maisons de planches groupées en villages et consacraient à la pêche la plus grande partie de leur activité. Ils possédaient une organisation sociale extrêmement élaborée. Le fait qu'ils fabriquaient des planches n'est pas l'indice de moyens techniques supérieurs à ceux des autres indigènes du pays: mais ils vivaient parmi les grands thuyas dont le bois se fend facilement au moyen de coins.

L'arrivée des Blancs devait modifier radicalement la vie aborigène. L'amérindien du Canada subit depuis une perpétuelle évolution. Au début de l'histoire coloniale, l'Algique courait la forêt, préoccupé des vivres et du vêtement de sa famille. Devenu 
trappeur, il constitue le premier chaînon d'un commerce à l'échelle mondiale. Ses territoires de chasses familiaux, tels qu'on les connaît aujourd'hui, doivent peut-être leur structure à cette transformation culturelle. La carabine a remplacé l'arc, les pièges de métal ceux de bois; la toile s'est substituée à l'écorce de bouleau dans le wigwam et le canot, le petit poêle de tôle, si léger, à l'ancien feu, ouvert au centre de la tente et exigeant une large ouverture dans le toit. La cuisine algique, basée sur la chasse, la pêche et la cueillette, gravite, maintenant autour du pain, - la banic (de l'écossais bannock), - et du thé. Au vêtement primitif, s'est substitué celui des Blancs, suivant les modes de l'époque. Des ethnologues ont cru voir une influence esquimaude dans la redingote des Naskapi, encore en usage au début du siècle. Les Esquimaudes, en effet, sont de très habiles couturières, mais la veste naskapi copie simplement le justaucorps français des XVIIe et XVIIIe siècles. L'indigène le plus primitif de l'est du pays il y a dix ans, le Naskapi, délaisse la chasse depuis l'exploration des mines de l'Ungava.

Le cheval, abandonné dans le désert du sud-ouest des ÉtatsUnis par les Espagnols et qui d'étape en étape rejoignit la prairie canadienne, permit une recrudescence de la chasse au bison. Le tipi primitif, en peau de cerf, s'adaptait au travois tiré par les chiens; le cuir de bison put servir à cette fin quand le cheval devint l'animal de trait. Après la disparition du bison, la cotonnade fait son apparition; mais alors, - trait historique important, - l'ouverture de la Prairie à la colonisation européenne a renfermé les indigènes dans des réserves, pour y vivre d'agriculture et de rentes de l'état, obtenues en échange des territoires tribaux. Les puits de pétrole transforment maintenant les Piedsnoirs en capitalistes. Les Micmacs, les Abénaquis, des peuplades de la côte du Pacifique se sont orientés, maladroitement et sans guides, vers l'agriculture. Les cultivateurs iroquois de Caughnawaga deviennent des ouvriers métallurgistes recherchés.

Avant même la conversion au christianisme, l'animisme primitif semble avoir subi une acculturation au contact des Blancs. L'idée d'un dieu suprême, intégré dans le panthéon animiste, est probablement une importation européenne qui, 
d'étape en étape, rejoignait parfois des tribus lointaines avant les missionnaires eux-mêmes et par le mode de transmission orale que l'on a nommé le Mocassin Telegraph.

La structure sociale des primitifs, adaptée au milieu et aux circonstances, faisait des tribus et des bandes des unités solides. L'autorité des chefs avait alors une signification ils étaient choisis à cause de leur compétence - confirmée par les joutes oratoires autour du feu de camp, où il fallait de toute nécessité dire des choses sensées. Les indigènes considéraient leur race avec fierté, au point que la plupart des noms qu'ils donnaient à leur groupe signifiaient "le peuple", les "vrais hommes". Ainsi, inuit, chez les Esquimaux, et ilnout (d'où Illinois), ou une forme apparentée chez les Algiques. Dans une tentative plus louable qu'adroite pour intégrer les indigènes dans la nation canadienne, on a saboté leur structure sociale sans leur donner les échelons dont ils avaient besoin pour passer d'une étape culturelle à l'autre. Trop souvent, on a réduit les descendants des fiers Amérindiens à des groupements marginaux dont on espère tout au plus, dans les meilleures conditions, tirer des manœuvres. Pourtant, le rôle qu'ils ont joué dans l'histoire canadienne permettait d'espérer davantage. L'exemple des Groenlandais est concluant.

\section{LA TRAME AMÉRINDIENNE DE L'HISTOIRE DU CANADA}

Les sagas islandaises, rédigées après des années de transmission orale, sont les premiers documents à mentionner les Amérindiens. Les Norsemen, qui fondèrent la colonie du Vinland, il y a près d'un millénaire, rencontrèrent des Skraelings, - peuple de Dorset, Esquimaux ou Béothuk, on ne sait, - et d'autres indigènes qu'ils durent affronter. Les témoignages imprécis laissent place aux conjectures, mais l'archéologue danois Nelgaard a découvert des traces d'occupation viking fort ancienne, qui fourniront des éléments certains sur l'histoire des établissements scandinaves et peut-être même l'identité des indigènes auxquels ils se heurtèrent. 
Cabot vit probablement des Béothuk. Des contemporains, à la suite, croisèrent sans doute des indigènes canadiens, mais il faut attendre le premier voyage de Cartier, en 1534, pour en arriver à de véritables contacts d'Européens et d'AmérindoCanadiens. Après la rencontre de Hurons-Iroquois dans la baie de Gaspé, il nous entretient de leurs coutumes et d'une plante cultivée, le maïs. Deux jeunes indigènes, Domagaya et Taignoagny, ramenés en France à leur corps défendant, deviendront l'année suivante les premiers interprètes canadiens à Stadaconé (Québec). Grâce à eux, le capitaine saura de Donnacona que le St-Laurent n'est pas un détroit vers la mer de Cathay, et accumulera de précieux renseignements qui permettront aux géographes $d u$ vieux monde de dresser les premières cartes $d u$ Canada. Rabelais s'est moqué, - non sans raison, - des fabuleux ouï-dire de Cartier, mais ils ne sont pas tous pour cela des inepties. Ces renseignements, - déformés par l'interprétation déficiente de Taignoagny et Domagaya et par la naïveté d'étrangers en quête de merveilleux, - ne sont pas toujours dénués de toute vérité. $\mathrm{Au}$ XVIIIe siècle, des naturalistes croyaient encore à l'existence de la licorne héraldique, parce qu'ils ignoraient son prototype, le narval. On comprend que la notion des unipèdes ait pu pénétrer dans le récit, si l'on songe que les Esquimaux, vêtus de l'anorak à longue queue, paraissent unijambistes à distance.

Pendant l'hiver de 1535-36, l'équipage de Cartier est en proie à une grave avitaminose, méconnue alors, le scorbut. La relation du voyageur présente une description élaborée des symptômes, - la première encore consacrée à la maladie, - qui ne déparerait pas un traité moderne de pathologie. Grâce aux conseils des interprètes, l'équipage moribond et fortement décimé se remet sur pied en quelques jours au moyen de la décoction d'un conifère, l'annedda, sur l'identité duquel on a longuement discuté, mais que des travaux récents (Jacques Rousseau, 1954) ont définitivement établie. Dès lors, le cèdre blanc (Thuja occidentalis) reçut le nom d'arbre de vie, conservé depuis dans la phytonymie anglaise (Arbor vitæ). 
$\mathrm{Au}$ premier rang des contributions de l'indigène à la vie européenne, se place son rôle dans la Découverte. Grâce à l'Amérindien, à son canot, ses raquettes et ses interprètes, issus des premières unions de Français et de femmes sauvages, Champlain, Jolliet et La Vérendrye se dirigent vers les "pays d'en haut" parfois sur de simples filets d'eau, et découvrent un continent.

Grâce aux chasseurs algiques aussi, Radisson et tous les coureurs des bois s'enfoncent dans la forêt boréale et créent un commerce à l'échelle mondiale, qui joue un rôle primordial dans l'histoire canadienne. La traite de la fourrure était venue s'intégrer dans la vie indienne en remplaçant les échanges préhistoriques d'écorce de bouleau, de coquillages, de pierres à pipes, de médecines et de charmes, qui se pratiquaient entre les tribus du continent, depuis les jours lointains de la préhistoire. Pendant que la forêt voyait surgir une nouvelle unité monétaire, - le castor, - le marché du wampum (tiré d'un coquillage, le Venus mercenaria), sous contrôle iroquois, sombrait dans le krach provoqué par l'importation des perles multicolores d'Europe. Ce troc des fourrures, longtemps plus important que l'agriculture, et à laquelle la Nouvelle-France dut sa survie, fit surgir de grandes villes, mais il eut aussi ses jours néfastes avec la contrebando de l'eau-de-vie et la guerre des fourrures.

La guerre de la fourrure débute, à l'époque de Champlain, entre le monopole hollandais de Fort-Orange et celui des CentAssociés à Québec, pour se terminer seulement deux siècles plus tard avec la fusion de la Compagnie de la Baie d'Hudson et de la Compagnie du Nord-Ouest. Elle a déterminé les alliances, déclanché les guerres indiennes et provoqué les incursions entre les colonies françaises et anglo-américaines, semant l'incendie, la destruction, les captivités, la levée des scalps et la torture au poteau. La guerre des fourrures, qui devait conduire les Iroquois jusqu'au lac Mistassini, reste après la maladie le premier facteur de dépopulation du nord-est amérindien. Toujours la chasse aux pelleteries poussera les coureurs des bois au centre du continent, où ils donneront naissance à la nation métisse, et fera rouler les Covered wagons vers le Pacifique, à la suite de 
Canadiens qui ont marqué de noms français les principaux jalons de la route de l'Ouest américain.

Les alliances indiennes ont exacerbé le conflit anglo-français, - né d'abord de divergences politiques européennes - et qui devait aboutir en Amérique en 1760 à la perte de la NouvelleFrance sur les plaines d'Abraham, puis en 1783, en NouvelleAngleterre, à l'indépendance américaine. Le conflit anglo-français a provoqué chez les indigènes des mouvements d'imigration loyaliste, celui des Abénaquis du Maine, sur le Saint-Laurent en 1700 , puis celui des Iroquois venus demander la protection du drapeau anglais, en Ontario, après la séparation des Etats-Unis.

Les instructions du Roi à Jacques Cartier mentionnent les deux motifs présidant aux voyages de découvertes: la découverte de richesses et la conversion des âmes païennes. Assurément, le gouvernement apporte une aide indubitable aux missionnaires; mais pour le néophyte, très souvent, l'attitude du pouvoir séculier et l'activité des Européens laissent du Blanc une image qui s'accorde assez mal avec l'enseignement missionnaire. Ce comportement bicéphale, - ce jeu de souque-à-la-corde spirituel, devait marquer définitivement la mentalité de l'indigène, déprécier ses protecteurs, retarder son intégration dans la civilisation et réduire sa contribution à la culture canadienne. Le pouvoir séculier, d'ailleurs, vit fréquemment dans le missionnaire un émissaire pour aplanir la voie aux missions commerciales. Il n'y a donc pas à s'étonner que la conversion soit rudimentaire et conduise à un dualisme religieux (Rousseau \& Rousseau, 1952) subsistant toujours dans des régions visitées par le missionnaire trois siècles plus tôt.

Le choc des cultures, situées aux pôles extrêmes, explique pour beaucoup ces heurts qui opposèrent le missionnaire et celui qu'il voulait faire bondir du paléolithique aux temps modernes. Sans vouloir minimiser en rien l'œuvre spirituelle et humaine de Brébeuf, de Jogues et de leurs collègues, il faut reconnaître que l'incompréhension devait fatalement engendrer la violence au pied de l'autel, et d'autant plus certainement que des agioteurs commerciaux, dans l'ombre, encourageaient les Sauvages à se 
libérer de chefs spirituels, trop enclins à freiner les ambitions démesurées. Quand Jogues vint pour la première fois chez les Iroquois avec le donné Guillaume Couture, une maladie contagieuse, - une grippe peut-être, — sévit pendant que le Jésuite, en tournée de mission, avait laissé un coffret au camp de base. Les shamans, déjà jaloux de cette compétition spirituelle inégale, virent dans le coffret l'esprit de la maladie, attendant le départ du maître pour sortir de sa prison et attaquer les victimes choisies par le "sorcier blanc". Et Jogues subit alors son premier martyre. Si l'on néglige de tabler sur cette incompréhension culturelle, il est impossible d'analyser correctement l'évolution de notre histoire.

La contribution de l'indigène à la vie moderne est immense. Le Blanc lui doit la raquette, la tobagane, le canot d'écorce, sans lesquels il n'aurait jamais conquis la forêt boréale. Et encore aujourd'hui, sans le canot de toile, fils du bouleau, explorateurs, forestiers, arpenteurs et prospecteurs ne réussiraient pas à franchir la forêt coniférienne.

Le tabac, - cultivé par les Hurons et les Iroquois et l'objet d'un commerce important entre ces derniers et les Algonkiens, - est devenu en quelque sorte l'une des caractéristiques de la vie moderne. Les jardins préhistoriques, en amont de Montréal, rassemblaient à côté du tabac, le maïs, la courge et le haricot, totalement inconnus en Europe avant la Découverte. Les Hurons tiraient du grand soleil l'huile pour lubrifier la chevelure. Le topinambour, découvert dans l'est du Canada par Samuel de Champlain, pénètre en France avec l'historien Marc Lescarbot. La pomme de terre, nommée patate au Canada et dans plusieurs provinces de France, vient du Pérou. Les Caraïbes ont fourni le manioc et la patate sucrée. Au Mexique, le piment constituait le principal condiment et un légume apprécié. Le coton se cultivait au Nouveau-Mexique. Et parmi les autres espèces, originaires du nouveau continent, mentionnons la tomate, le cacao, l'arachide, le papayer, l'ananas, l'avocatier et l'arrow-root. Les indigènes de l'Amérique centrale et de l'Amérique du sud, à eux seuls, fournirent au-delà de cent plantes à l'agriculture mondiale, transformant ainsi la cuisine et l'industrie du vieux monde. 
Les Amérindiens du Canada connaissaient probablement le sirop d'érable, mais pas le sucre cristallisé, le "sucre du pays". De rares recettes indigènes ont pris place dans la cuisine eurocanadienne, comme le blé d'Inde lessivé, les autres apprêts du maïs, le succotash, - un mélange de grains de blé d'Inde et de haricots bouillis, très populaire en Nouvelle-Angleterre, - le gibier au riz sauvage, enfin des techniques de panification qui se sont perfectionnées dans la forêt boréale pour le plus grand profit des explorateurs modernes.

Les langues anglaise et française ont emprunté aux dialectes amérindiens. Pow-wow, canoe, tepee, chipmunk, moose, hominy, squash, tamarack, et d'autres en anglais. En français, pas moins de cent mots dont canot, tobagane, nâgane, kometik, wigwam et plus de vingt-cinq noms de plantes et d'animaux, dans la province de Québec seulement.

Le premier sauvage à jouer un rôle dans l'histoire et dont nous ayons conservé le nom, Donnaconna, rencontre Jacques Cartier à Gaspé en 1534. Ses neveux, Teignoagny et Domagaya, ouvrent la liste des interprètes euro-amérindiens. Quelques années plus tard, vers 1570 , le Huron Dekanawida fonde la ligue iroquoise, - dans laquelle refusent d'entrer les Hurons, - avec l'aide de son disciple, le Mohawk Hiawatha, dont s'est emparé la légende. Chez les Micmacs, en 1611, les Français rencontrent le chef Membertou. Entre 1636 et 1644, en pays attikamek, le shaman Étienne Pigarouich se convertit au catholicisme, puis apostasie, pour recommencer avec virtuosité ses professions de foi à bascule. Le combat de Dollard, au bord du Long-Sault, en 1660, consacre l'héroïsme du chef huron Annahotoha et du chef algonquin Métigonèque. En 1677, Garagonthié préside aux CinqNations, pendant que la petite mohawk, Kateri Tekakwita (165680 ), achève à la Prairie de la Madeleine sa courte existence vouée au mysticisme. Kondiaronk, dit le Rat, un chef huron de Michillimakinac, en 1690, joue le rôle peu glorieux d'agent provocateur ou d'agioteur.

Les guerres permettent à l'Outaouais Pontiac (1720) et au Shawnee Tecumseh (1812) de s'illustrer. Handsome Lake, se 
réformant après une vie mouvementée, propose aux Iroquois une nouvelle religion née d'un syncrétisme de l'animisme et du christianisme. Nancy Shanawdithit s'illustre passivement, en 1829, en mourant en captivité à Terre-Neuve, - dernier survivant des vrais Peaux-rouges, les Béothuk. Enfin, le chef sioux Sitting Bull (1834-1890), rebelle irréductible de l'ouest américain, finit assassiné, après avoir bénéficié un temps du droit d'asile au Canada. D'autres noms aussi mériteraient mention.

Moins de cinquante noms Amérindiens dans nos annales, aux XVIe et XVIIe siècles! mais ce serait méconnaître l'histoire de la civilisation que d'y voir la seule contribution du monde sauvage. Humbles artisans de l'acculturation, héros obscurs tombés au cours des combats, interprètes et hommes de canot, qui ont halé tout le continent vers la vie moderne, c'est à cette masse anonyme que l'histoire doit plusieurs de ses plus belles pages.

Centre d'Études nordiques,

JACQUes Rousseau

Université Laval, Québec.

\section{BIBLIOGRAPHIE}

On ne trouvera ici qu'une liste sommaire des ouvrages relatifs au sujet et notamment les ouvrages cités. Les travaux de l'auteur, cités à la suite, renfermant souvent des bibliographies élaborées, suppléeront à cette déficience.

HoDGE, Frederick Webb. [Editeur]. Handbook of American Indians North of Mexico. 2 vol., 972 et 1221 pp., 1907. Bur. Amer. Ethnology, Bull. 30.

Howley, James P. The Beothuks or Red Indians, the aboriginal inhabitants of Newfoundland. 348 pp. Cambridge (University Press), 1915.

Jenness, Diamond. Indians of Canada. 452 pp., 1958. Nat. Museum of Canada, Bull. 65. (Première édition, 1932).

LEE, Thomas E. The question of Indian Origins. Science of Man, Vol. 1 : 159-167, 1961.

RIDLEY, Frank. Transatlantic of primitive man: Eastern Canada and Northwestern Russia. Pennsylvania Archaeologist, 30: $46-57,1960$. 
Rousseau, Jacques. “A travers l'Ungava”, L'actualité économique, 25: 83-131, 1949.

- "Persistances païennes chez les Amérindiens de la forêt boréale”, Cahiers des Dix, 17: 183-208, 1952.

- "Rites païens de la forêt québécoise: la tente tremblante et la suerie", Cahiers des Dix, 18: 129-155, 1953.

- "L'annedda et l'arbre de vie", Revue d'Histoire de l'Amérique francaise, 8 : 171-212, 1954.

- "Des menus rites païens de la forêt canadienne", Cahiers des Dix, $19: 187-232,1954$.

- "L'origine et l'évolution du mot "Esquimaux"," Cahiers des Dix, 20: 179-198, 1955.

- "Les américanismes du parler français au Canada", Cahiers des Dix, 21: 89-104, 1956.

- "L'Indien de la forêt boréale, élément de la formation écologique", in Royal Society of Canada, Studia varia, pp. 37-51, 1957.

- "Astam Mitchoun ! Essai sur la gastronomie amérindienne", Cahiers des Dix, 22: 193-211, 1957.

- "Ces gens qu'on dit Sauvages", Cahiers des Dix, 23: 53-90, 1958.

- "Les Sachems délibèrent autour du feu de camp", Cahiers des Dix, 24 : 9-49, 1959.

- "Les premiers Canadiens", Cahiers des Dix, 25: 9-64, 1960. Ces trois dernières études constituent un tout.

- "La trame forestière de l'histoire canadienne", Cahiers des Dix, $26: 17-54,1961$.

- "Le dernier des Peaux-Rouges", Cahiers des Dix, 27: 47-76, 1962.

Rousseau, Madeleine et Jacques, "La crainte des Iroquois chez les Mistassins", Revue d'Histoire de l'Amérique française, $2: 13-26,1948$.

- "Le dualisme religieux des peuplades de la forêt boréale". In Selected Papers of the international Congress of Americanists, 2: 118-126, 1952.

SPECK, Frank G., Naskapi: The savage hunters of the Labrador Peninsula. 248 pp., Norman (Oklahoma), 1935.

Swanton, John R., The Indian tribes of North America. Bull. 145, Bureau of American Ethnology, Washington, 1952. 